\title{
Delay Reduction in Multi-Hop Device-to-Device Communication using Network Coding
}

\author{
Ahmed Douik, Student Member, IEEE, Sameh Sorour, Senior Member, IEEE, Tareq Y. Al-Naffouri, Member, \\ IEEE, Hong-Chuan Yang, Senior Member, IEEE, and Mohamed-Slim Alouini, Fellow, IEEE
}

\begin{abstract}
This paper considers the problem of reducing the broadcast decoding delay of wireless networks using instantly decodable network coding (IDNC) based device-to-device (D2D) communications. In contrast with previous works that assume a fully connected network, this paper investigates a partially connected configuration in which multiple devices are allowed to transmit simultaneously. To that end, the different events occurring at each device are identified so as to derive an expression for the probability distribution of the decoding delay. Afterward, the joint optimization problem over the set of transmitting devices and packet combination of each is formulated. The optimal solution of the joint optimization problem is derived using a graph theoretic approach by introducing the cooperation graph in which each vertex represents a transmitting device with a weight translating its contribution to the network. The paper solves the problem by reformulating it as a maximum weight clique problem which can efficiently be solved. Numerical results suggest that the proposed solution outperforms state-of-the-art schemes and provides significant gain, especially for poorly connected networks.
\end{abstract}

Index Terms-Instantly decodable network coding, Device-todevice, Partially connected network, Maximum weight clique.

\section{INTRODUCTION}

Network Coding $(N C)$ is a promising technique [2] to significantly improve the throughput, i.e., network capacity, and to minimize delay over wireless erasure channels. These benefits are of keen interest for the proliferation and spread of real-time applications as they require quick and reliable packet transmission over lossy channels with low latency [3], such as streaming, cellular, satellite networks, and Internet television.

Two classes of NC can be distinguished in the literature, namely the Random Network Coding (RNC) [4], [5] and the Opportunistic Network Coding (ONC) [6], [7]. While the sender in RNC combines packets using random coefficients, ONC exploits the different received and lost packets to encode packets in real-time. Although RNC is optimal in reducing the number of transmissions even without feedback, it is not suitable for real-time applications as it does not support progressive decoding of the frame. Moreover, unlike ONC,

A part of this paper [1] appears in proc. of IEEE International Symposium on Network Coding (NetCod' 2015), Sydney, Australia.

Ahmed Douik is with the Department of Electrical Engineering, California Institute of Technology, Pasadena, CA 91125 USA (e-mail: ahmed.douik@caltech.edu).

Sameh Sorour is with the Department of Electrical and Computer Engineering, University of Idaho, Moscow, ID 83844, USA (e-mail: samehsorour@uidaho.edu).

T. Y. Al-Naffouri and M.-S. Alouini are with the Division of Computer, Electrical and Mathematical Sciences and Engineering, King Abdullah University of Science and Technology, Thuwal 23955-6900, Saudi Arabia (e-mail: \{tareq.alnaffouri,slim.alouini\}@ kaust.edu.sa).

Hong-Chuan Yang is with the Department of Electrical and Computer Engineering, University of Victoria (UVIC), British Columbia, Canada, email: hy@uvic.ca. the decoding computation complexity of RNC is prohibitive for real-time applications on battery-powered devices.

This paper is interested in delay sensitive broadcast services, in which each device should receive all packets within a frame with minimum delay [8]. A suitable technique for such applications is the Instantly Decodable Network Coding (IDNC) [9]. In this subclass of ONC, the sender encodes the packets using binary XOR and devices decode them by the same means, which is an important property that ensures fast encoding/decoding and overcomes the computationally expensive matrix inversion operations. Such low-computation property makes IDNC a suitable technology for batterypowered devices. In addition, non-instantly decodable packets are not stored, which eliminates the need for buffers and allows the design of cost-efficient receivers. Thanks to its numerous benefits, IDNC was a subject of intensive research in the past few years [8]-[22]. A survey on the recent advantaces in IDNC schemes and application is available in [23].

The commonly used performance metrics in IDNC are the completion time and the decoding delay. The former definition considers the performance as the overall transmission time and the latter as the individual delays when receiving a packet that does not bring new information at its reception instant. This paper considers the minimization of the decoding delay as it represents a crucial step to study the completion time reduction, e.g., [24].

\section{A. Related Work}

Determining the packet combinations for the whole recovery phase to optimally reduce the decoding delay is shown to be intractable even for erasure-free communication [25] or offline studies [26], i.e., erasure events are known in advance. In order to overcome the complexity, the commonly adopted approach is to examine the online decoding delay in which the delay is minimized at each transmission slot.

In all aforementioned works, the wireless centralized sender of a Point to Multi-Point (PMP) network, such as cellular, Wi$\mathrm{Fi}$, and roadside to vehicle systems, is the only transmitter in charge of both the packet sending and recovery processes. This approach consumes a lot of the sender resources and threats its ability to deliver the packets with the desired rates. The problem is expected to further escalate in the next generation mobile radio systems $(5 \mathrm{G})$ as the data rates and the Quality of Service (QoS) requirements are becoming even more constraining. The notion of Device-to-Device (D2D) communication is a suitable technique to overcome the problem [27]. In a D2D configuration, devices exchange packets with each other over short-range and possibly more reliable channels. Unlike traditional D2D underlying or overlying a cellular 
network, this paper is interested cooperative data exchange D2D networks, e.g., fast and secure data communications over ad-hoc networks. Furthermore, the technology can be combined with green communication algorithms to enable highly power-efficient communications [28], [29].

In PMP systems, the optimization is performed only over on the packet combination to be transmitted. However, the optimization in a D2D environment should be also made on the set of transmitting devices in every transmission to achieve the best network performance. Aboutorab et al. [30] investigate the problem of minimizing the sum decoding delay in a centrally controlled D2D environment, wherein a leader, in this case the cellular BS, takes all decisions. The authors in [31] extend the study to the imperfect feedback D2D networks. In a fully distributed D2D system, [32], [33] considers the completion time and decodind delay minimization, respectively, using game theory as a tool to improve the distributed solution.

These prior works on IDNC-based D2D systems assume that the network is fully connected (FC-D2D), i.e., each device can target all other devices over one-hop transmission. Therefore, only one device can transmit at each transmission slot. This assumption of FC-D2D may not apply in some realistic scenarios due to the short transmission ranges of devices and their widespread over a large cell area. Furthermore, longer-range D2D transmissions can easily limit the desirable property of more reliable communications between devices compared to those from the centralized sender. This work proposes to study the decoding delay minimization in the case of partially connected D2D networks (PC-D2D). The partially connected configuration adds a new dimension to the problem as many devices can communicate simultaneously, each with a different packet combination.

\section{B. Contribution}

The main contribution of this paper is to study the reduction of the decoding delay using IDNC in partially connected D2D networks. Unlike fully connected D2D networks, in partially connected systems the problem of selecting the transmitting devices and the problem of selecting the packet combinations are inter-dependent. Therefore, the optimization problem should be addressed jointly. Although the paper focuses on a centrally controlled networks, the decoding delay analysis presented in this document serves as a reference to future works on fully distributed systems as it provides a lower bound on the achievable decoding delay.

The paper first identifies the different expected decoding delays for each device in the network. These expressions are exploited to formulate the minimum decoding delay problem in IDNC-based PC-D2D networks. The paper addresses the decoding delay reduction by introducing both local IDNC packet generation graphs and a D2D cooperation graph. The problem is reformulated as a maximum weight clique search that can be efficiently solved using existing literature from graph theory [34], [35]. Both collision-free schedules, i.e., transmission schedules not allowing a device to be in the range of two simultaneously transmitting devices, and collisionallowed schedules are be studied this paper. Simulation results show that the proposed solution displays substantial gains as compared with the fully connected D2D network approach and the PMP configuration.

The rest of this paper is organized as follows: Section II introduces the system model and parameters. The decoding delay minimization problem in partially connected D2D network is formulated in Section III. Section IV solves the problem in the particular collision-free case. In Section V, the decoding delay minimization problem is solved. Simulation results are illustrated and discussed in Section VI before concluding in Section VII.

\section{SyStem Model AND PARAMETERS}

Consider a wireless base-station (BS) that desires to transmit $N$ different packets to a set $\mathcal{M}$ of $M$ devices. Let $\mathcal{N}$ denotes the frame consisting of the various source packets. The BS begins by broadcasting the $N$ source packets sequentially. Each device listens and sends an acknowledgment upon each successful reception. The probability of a packet loss at device $i$ when the BS is transmitting is $q_{i}, \forall i \in \mathcal{M}$. At the end of this initial phase, we assume that each packet of the frame $\mathcal{N}$ is acknowledged by at least one device. Otherwise, the BS keeps broadcasting the packet until the condition is verified. After the initialization phase, the packets of the frame can be in one of the following two sets for each device $i$ :

- The Has set $\mathcal{H}_{i}$ : the set of packets received by device $i$.

- The Wants set $\mathcal{W}_{i}$ : the set of packets lost by device $i$.

In the recovery phase, devices cooperate using IDNC to ensure that everyone successfully receives all $N$ packets. Exploiting the asymmetry of the side information of all users, a subset of them transmit, simultaneously and using the same radio resource, XOR combinations of the source packets, with the aim to maximize the number of users capable of decoding a new source packet from these XOR combinations. In this phase, the coded packets can be one of the following options for device $i$ :

- Non-innovative: A packet is non-innovative for device $i$ if it does not contain any packet from $\mathcal{W}_{i}$.

- Instantly Decodable: A packet is instantly decodable for device $i$ if it contains exactly one packet from $\mathcal{W}_{i}$.

- Non-Instantly Decodable: A packet is non-instantly decodable for device $i$ if it contains more than one packet from $\mathcal{W}_{i}$.

Definition 1. At any recovery phase transmission, a device $i$, with non-empty Wants sets, experiences one unit increase of decoding delay if he cannot hear exactly one transmission or if it hears a packet that is either non-instantly decodable or non-innovative.

Therefore, the decoding delay is a measure of the number of non-useful transmissions that are successfully received. Let $\mathcal{C}_{i}$ be the coverage zone of device $i$ defined as the set of devices in the transmission range of device $i$ and let $\mathbf{P}=\left[p_{i j}\right], \forall(i, j) \in \mathcal{M}^{2}$ denote the packet erasure probability from device $i$ to device $j$. Since users transmit simultaneously in the same frequency band, devices in the intersection of 
the coverage zone of multiple transmitting device experience collision and are not able to retrieve any useful information.

The notation $\bar{X}$, where $X$ is a set in the ensemble $E$, refers to the complementary of the set, i.e., $\bar{X}=E \backslash X$. The notation $\mathcal{P}(E)$ refers to the power set of the ensemble $E$. In other words, $\mathcal{P}(E)$ is the set of all subsets of the ensemble $E$. A partition $\left\{X_{i}\right\}_{1<i<n}$ of the ensemble $E$ is denoted by $E=$ $\bigoplus_{i=1}^{n} X_{i}$. Let $|\bar{X}|$ denote the cardinality of the set $X$.

\section{Minimum Decoding Delay Problem FORMULATION}

This section first formulates the minimum decoding delay problem in partially connected D2D networks. The problem is expressed as a joint optimization over the set of transmitting devices and the packet combinations to be transmitted. Further, the section illustrates the optimal solution for a fixed set of transmitting devices by introducing the local IDNC graph for packet generation.

\section{A. Problem Formulation}

Let $\mathcal{A} \in \mathcal{P}(\mathcal{M})$ be the set of transmitting devices and let $\mathcal{T}(\mathcal{A})$ be the set of non-transmitting devices in collision. In other words, $\mathcal{T}(\mathcal{A})$ is the set of devices that can hear multiple transmissions from the set of transmitting devices $\mathcal{A}$. The mathematical definition of this set is:

$\mathcal{T}(\mathcal{A})=\left\{i \notin \mathcal{A} \mid \exists(m, n) \in \mathcal{A}^{2}, m \neq n, i \in \mathcal{C}_{n} \cap \mathcal{C}_{m}\right\}$ Define $\mathcal{S}(\mathcal{A})$ as the set of devices that are not in the transmission range of any transmitting device. Formally, the set is written as:

$$
\mathcal{S}(\mathcal{A})=\left\{i \in \mathcal{M} \mid \nexists j \in \mathcal{A}, i \in \mathcal{C}_{j}\right\} .
$$

Define the opportunity zone $\mathcal{O}_{i}(\mathcal{A})=\mathcal{C}_{i} \backslash(\mathcal{A} \cup \mathcal{T}(\mathcal{A}))$ as the set of devices that can be targeted by device $i$ and can decode a packet from the transmission. Let $M_{w}$ be the set of devices having non-empty Wants set and let $\kappa_{i}(\mathcal{A})$ be the packet combination to be transmitted by device $i \in \mathcal{A}$. Define $\tau_{i}\left(\kappa_{i}(\mathcal{A})\right)$ as the set of devices that can decode the packet combination $\kappa_{i}$ sent by device $i$, i.e., :

$$
\tau_{i}\left(\kappa_{i}(\mathcal{A})\right)=\left\{j \in \mathcal{C}_{i}|| \kappa_{i} \cap \mathcal{W}_{j} \mid=1 \text { and } j \notin \mathcal{T}(\mathcal{A})\right\} .
$$

Remark 1. The variables defined above should be all a function of the set of transmitting devices $\mathcal{A}$. However, for notation convenience, the set will be dropped unless it is required, e.g., we will use $\mathcal{T}$ and $\tau_{i}\left(\kappa_{i}\right)$ instead of $\mathcal{T}(\mathcal{A})$ and $\tau_{i}\left(\kappa_{i}(\mathcal{A})\right)$.

This paper is interested in selecting the set of transmitting devices and their packet combinations so as to minimize the expected decoding delay increase. Let $D(\mathcal{A}, \kappa)$ be the total decoding delay increase, the paper solves the following problem:

$$
\min _{\substack{\mathcal{A} \in \mathcal{P}(\mathcal{M}) \\ \kappa_{i}(\mathcal{A}) \in \mathcal{P}\left(\mathcal{H}_{i}\right)}} \mathbb{E}[D(\mathcal{A}, \kappa)] .
$$

Using Definition 1, the decoding delay reduction problem (3) can be reformulated using the system parameters.
Proposition 1. The decoding delay reduction problem in partially connected D2D network can be formulated as follows:

$$
\begin{aligned}
& \max _{\mathcal{A} \in \mathcal{P}(\mathcal{M})}-\left|\mathcal{A} \cap M_{w}\right|-\left|\mathcal{T} \cap M_{w}\right|-\left|\mathcal{S} \cap M_{w}\right| \\
& \quad+\sum_{i \in \mathcal{A}}\left(\sum_{j \in \tau_{i}\left(\kappa_{i}^{*}\right)} 1-p_{i j}\right),
\end{aligned}
$$

with the packet combinations being given by:

$$
\kappa_{i}^{*}=\arg \max _{\kappa_{i} \in \mathcal{P}\left(\mathcal{H}_{i}\right)}\left(\sum_{j \in \tau_{i}\left(\kappa_{i}\right)} 1-p_{i j}\right), \forall i \in \mathcal{A} .
$$

Proof. In order to formulate the decoding delay minimization problem in partially connected IDNC-based D2D networks, the expected decoding delay increase of each device is first expressed. Afterward, these expressions are used to formulate the problem as a joint optimization over the set of transmitting devices and the packet combinations to be transmitted. The complete proof can be found in Appendix A.

Both optimization problems (4) and (5) are inter-dependent and cannot be solved separately. In the next subsection, we derive the optimal packet mix for a fixed set of transmitting devices, i.e., the optimal $\kappa_{i}^{*}, i \in \mathcal{A}$ for a fixed set $\mathcal{A}$. Afterward, the complexity of finding the optimal solution of (4) for any reasonably sized network is discussed.

\section{B. Local IDNC Graphs for Packet Generation}

To solve the optimization problem (5), this subsection relies on a graph theoretical model first introduced in the context of PMP networks in [15]. Therefore, this section first extends the IDNC graph to account for the restrictions in packet possessions of the different devices and the partial connectivity of the graph. In this context, let such graph for each device be called its local IDNC graph. Finally, the problem (5) is reformulated as a maximum weight clique problem in the local IDNC graph.

In contrast with the PMP model that permits the generation of all packet mixes, in a D2D environment, each device can generate coding combinations only from the packets it possesses, i.e., packets in his Has set. Further, while the sender in the fully connected network can broadcast packets to all devices, each device in partially connected systems can target only devices in its transmission range. This subsection illustrates how the different devices can build their local IDNC graphs.

To construct the local IDNC graph $\mathcal{G}_{i}\left(\mathcal{V}_{i}, \mathcal{E}_{i}\right)$ of device $i \in$ $\mathcal{A}$, a vertex $v_{k l} \in \mathcal{V}_{i}$ is generated for each packet $l \in\left(\mathcal{W}_{k} \cap\right.$ $\left.\mathcal{H}_{i}\right), \forall k \in \mathcal{O}_{i}$. An edge in $\mathcal{E}_{i}$ connecting vertices $v_{k l}$ and $v_{m n}$ is created if one of the two following conditions is true:

- $l=n \Rightarrow$ Packet $l$ is needed by both devices $k$ and $m$.

- $l \in \mathcal{H}_{m}$ and $n \in \mathcal{H}_{k} \Rightarrow$ The packet combination $l \oplus n$ is instantly decodable for both devices $k$ and $m$.

The following lemma characterizes the solution of the decoding delay reduction problem (5) for a fixed set of transmitting devices $\mathcal{A}$.

Lemma 1. The optimal solution $\kappa_{i}^{*}$ of the optimization problem (5) for device $i \in \mathcal{A}$ is the maximum weight clique in the 


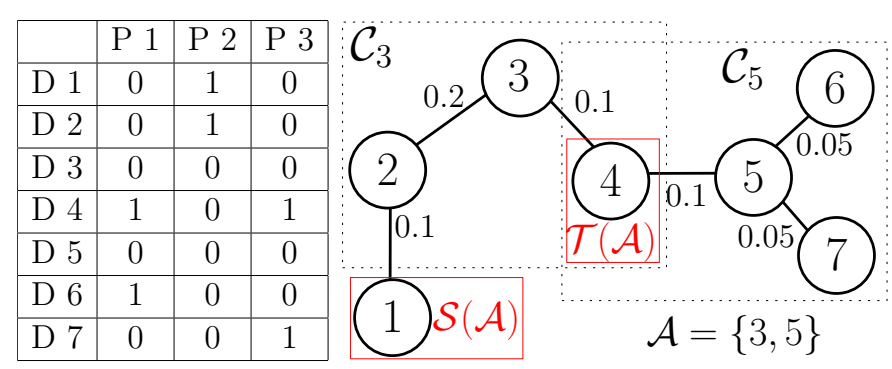

Fig. 1. Network composed of 7 devices and 3 packets. The feedback matrix represents the distribution of lost (1) and received packets (0) at each device. The erasure probabilities between devices is presented on the edges.

local IDNC graph $\mathcal{G}_{i}$ of device $i$ in which the weight of each vertex $v_{k l}$ is $1-p_{i k}$.

Proof. The proof of this lemma relies on the existence of a one-to-one mapping between every possible packet combinations and the cliques in the local IDNC graph. Such a mapping is demonstrated in [22] for a PMP network. In order to obtain a similar result for the configuration under investigation, the proof establishes a correspondence between the local IDNC graph for the $i$-th device and a PMP network with a reduced set of devices and packets. The complete proof can be found in Appendix B.

\section{Complexity of Finding the Optimal Solution}

Given the solution to the decoding delay reduction problem (5) for a fixed set of transmitting devices, the global optimal solution to the decoding delay reduction problem (4) can be obtained by solving problem (5) for all possible set of transmitting devices. Using an exhaustive search over the $M$ devices, the complexity is of the order of $2^{M} \mathfrak{f}$, where $\mathfrak{f}$ is the complexity of solving (5) that depends on the clique search algorithm. For example, $\mathfrak{f}=1.212^{M N}$ for the clique algorithm in [36]. Clearly, such approach is unfeasible for any reasonably sized network. The rest of the paper develops a tractable method for reaching the global optimal solution of the optimization problem in (4).

\section{Collision-Free CoOperative Solution}

This section presents the solution to the optimization problem in (4) under a collision-free restriction, namely $\mathcal{T}=\varnothing$. In other words, the problem is solved under the constraint that cooperation is allowed only when no device is subject to collision. The section shows that under the above condition, both problem variables become separable which allows reformulating the problem as a clique search in a newly designed cooperation graph. The optimal solution to the problem is reached in Section V by means of clustering devices and using both the cooperation and local IDNC graphs.

\section{A. Source of Tractability of the Collision-free Solution}

Consider the collision-enabled example shown in Fig. 1, which presents a system of 7 devices and 3 packets, with their connectivity pattern and the feedback matrix. Given the network configuration and the distribution of the lost/received packets, it can be clearly seen that only device 3 and device 5 can transmit a packet and ensure that it can be decoded by at least one device upon successful reception. When only device 3 is transmitting, the optimal solution is to target device 4 with packet 1 or 3 . When only device 5 is sending, the optimal solution is to target both devices 6 and 7 with the packet combination $1 \oplus 3$. However, when both devices 3 and 5 are sending, device 4 will be subject to collision. In this case, the optimal joint solution for both transmitting devices is that:

- device 3 targets device 2 with packet 2 .

- device 5 targets devices 6 and 7 with the combination $1 \oplus 3$.

The above example shows the high inter-dependence between the set of transmitting devices, transmitted packets and the possible devices that can benefit from them. This makes the problem variables non-separable. In this section, the decoding delay problem is relaxed by focusing on cooperation without collision. In other words, the cooperation between devices is allowed only when no device experiences collision. As a consequence, the modified problem is more mathematically tractable as it allows decoupling the variables. Hence, this section solves the following optimization over the set of transmitting devices:

$$
\max _{\mathcal{A} \in \mathcal{P}(\mathcal{M})}-\left|\mathcal{A} \cap M_{w}\right|-\left|\mathcal{S} \cap M_{w}\right|+\sum_{i \in \mathcal{A}}\left(\sum_{j \in \tau_{i}\left(\kappa_{i}^{*}\right)} 1-p_{i j}\right)
$$

subject to $\mathcal{T}=\varnothing$.

\section{B. Reformulated Problem}

The newly introduced constraint $\mathcal{T}=\varnothing$ limits the potential combinations of transmitting devices so as to avoid collision. Let $\mathcal{I}$ be the set of possible combinations of devices that satisfy the constraint. This set can be expressed as follows:

$$
\mathcal{I}=\left\{\mathcal{A} \in \mathcal{P}(\mathcal{M}) \mid \mathcal{C}_{i} \cap \mathcal{C}_{j}=\varnothing, \forall(i, j) \in \mathcal{A}^{2}\right\} .
$$

The following lemma reformulates the decoding delay reduction problem under collision-free cooperation.

Lemma 2. The decoding delay reduction problem in an collision-free IDNC-based D2D network (6) can be expressed as:

$$
\mathcal{A}^{*}=\arg \max _{\mathcal{A} \in \mathcal{I}}-\left|\mathcal{A} \cap M_{w}\right|-\left|\mathcal{S} \cap M_{w}\right|+\sum_{i \in \mathcal{A}} y_{i}\left(\kappa_{i}^{*}\right) .
$$

where

$$
y_{i}\left(\kappa_{i}^{*}\right)=\max _{\kappa \in \mathcal{G}_{i}}\left(\sum_{j \in \tau_{i}(\kappa)} 1-p_{i j}\right) .
$$

Proof. This proof establishes the independence of the set of transmitting devices and their packet combination given the collision-free constraint. Such conclusion is reached by showing that under the collision-free constraint, the localIDNC graph of any transmitting user does not depend on the other transmitting device. Therefore, using the result of Proposition 1, one concludes the decoupling of the optimization problems (4) and (5). Finally, expanding the formulation of each problem allows to obtain the desired result. The complete proof can be found in Appendix C. 
As hinted in (8) and (9), the constraint $\mathcal{T}=\varnothing$ allows the decoupling of the variables $\mathcal{A}$ and $\kappa_{i}, i \in \mathcal{A}$. Clearly, the optimization problem (9) is equivalent to the optimization problem (5) that can be solved using the local IDNC graphs. In the next subsection, the problem of selecting the set of transmitting devices (8) is solved using another graph-theoretic formulation.

\section{Solution using Cooperation Graph}

In general, two devices can transmit simultaneously if their coverage zones are mutually disjoint. Such combinations between the devices can be represented in a graph model called herein the cooperation graph. The graph is constructed by creating a vertex $v_{i}$ for each device in the network. Two vertices $v_{i}$ and $v_{j}$ are connected by an edge if their coverage zone are disjoint. In other words, they are connected if $\mathcal{C}_{i} \cap \mathcal{C}_{j}=\varnothing$. Using this graph, the following theorem characterizes the optimal solution of (8).

Theorem 1. The optimal solution to the reformulated decoding delay reduction problem in an collision-free IDNC-based $D 2 D$ network (6) is the maximum weight clique problem over the cooperation graph, in which the weight $\omega_{i}$ of each vertex $v_{i}$ is:

$$
\omega_{i}=\left|\mathcal{C}_{i} \cap M_{w}\right|-\left|\{i\} \cap M_{w}\right|+y_{i}\left(\kappa_{i}^{*}\right),
$$

where $\kappa_{i}^{*}$ is the solution to the maximum weight clique problem in the local IDNC graph of device i illustrated in Lemma 1.

Proof. To prove this theorem, we first reformulate the optimization problem (8) in a more tractable form. Afterward, we show that there is a one-to-one mapping between the set of feasible transmitting devices and the cliques in the cooperation graph. Finally, since the weight of the clique is equivalent to the objective function of (8) then the optimal solution of (8) is the maximum weight clique in the cooperation graph. The complete proof can be found in Appendix D.

\section{Clustering-Based Decoding Delay Reduction}

This section proposes to solve the decoding delay minimization problem (4) by introducing a clustering mechanism for devices and using the tools developed in the previous sections. The fundamental concept for solving problem (4) is to generate groups of non-interfering devices, called herein clusters, and to extend the cooperation graph formulation. As these clusters are non-interfering by construction, the results of the previous sections hold and can be used to find the optimal solution for (4). Therefore, this section first shows the mechanism for constructing such clusters. Afterward, the optimization problem in (4) is shown to be equivalent to a maximum weight clique problem that can be efficiently solved using existing literature from graph theory [34], [35].

\section{A. Cluster Generation}

This subsection illustrates the construction of a set $\mathcal{Z}$, called herein a clustering, that uniquely partition a set of transmitting devices $\mathcal{A} \in \mathcal{P}(\mathcal{M})$. Afterwards, Each cluster $Z \in \mathcal{Z}$ is represented by a vertex in the full cooperation graph. Finally, the uniqueness of the clustering $\mathcal{Z}$ enables the reformulation of the problem in the next subsections. Let $\mathcal{Z}$ be a partition of $\mathcal{A}$ such that:

1) All coverage zones of clusters $Z \in \mathcal{Z}$ are pairwise disjoint.

2) Within the same cluster $Z \in \mathcal{Z}$, each subset of devices is interfering with at least another device.

The mathematical definition of such a clustering $\mathcal{Z}$ is:

$$
\begin{gathered}
\bigoplus_{Z \in \mathcal{Z}} Z=\mathcal{A} \\
\mathcal{C}^{T}(Z) \cap \mathcal{C}^{T}\left(Z^{\prime}\right)=\varnothing, \forall Z \neq Z^{\prime} \in \mathcal{Z} \\
\mathcal{C}^{T}(z) \cap \mathcal{C}^{T}(Z \backslash z) \neq \varnothing, \forall z \subset Z, Z \in \mathcal{Z},
\end{gathered}
$$

where $\mathcal{C}^{T}(X)$ is the total coverage zone of all devices in the set $X$ defined as $\mathcal{C}^{T}(X)=\bigcup_{x \in X} \mathcal{C}_{x}$. The following proposition states the uniqueness of the clustering $\mathcal{Z}$ for any set of transmitting devices $\mathcal{A}$.

Proposition 2. For any combination of transmitting devices $\mathcal{A}$, there exists a unique clustering $\mathcal{Z}$ satisfying the constraints (11a), (11b) and (11c) simultaneously.

Proof. In order to establishes the uniqueness of the decomposition, this proof first show the existence of the decomposition by suggesting a sequential constructing method. The proposed algorithm construct a new cluster of each vertex that does not satisfy all conditions (11a), (11b) and (11c). The second part of the proof shows that any other partition that satisfy all constraints can be reduced by means of permutations to the one reached by the proposed algorithm. In other words, the partition is unique. The complete proof can be found in Appendix E.

\section{B. Full Cooperation Graph}

In this subsection, the cooperation graph is extended to include the previously defined clusters. The fundamental concept of constructing such a graph is to preserve all benefits of the cooperation graph while allowing all possible combinations of transmitting devices. This goal is reached by generating particular clusters and then connecting each pair of clusters that are not interfering with each other. Let $\mathbf{Z}$ be the set of clusters satisfying the constraint (11c) defined as follows:

$$
\mathbf{Z}=\left\{Z \in \mathcal{P}(\mathcal{M}) \mid \mathcal{C}^{T}(z) \cap \mathcal{C}^{T}(Z \backslash z) \neq \varnothing, \forall z \subset Z\right\}
$$

The full cooperation graph is constructed by generating a vertex $v$ for each cluster $Z \in \mathbf{Z}$. Two vertices $v$ and $v^{\prime}$ representing the clusters $Z$ and $Z^{\prime}$ are connected if they are non-interfering clusters. In other words, they are connected if they satisfy constraint (11b):

$$
\mathcal{C}^{T}(Z) \cap \mathcal{C}^{T}\left(Z^{\prime}\right)=\varnothing
$$

Let $\mathfrak{Z}$ be the set of cliques in the full cooperation graph. The following proposition links the set of cliques to the set of transmitting devices.

Proposition 3. There exists a one-to-one mapping between the set $\mathcal{P}(\mathcal{M})$ and $\mathfrak{Z}$. In other words, for each $\mathcal{A} \in \mathcal{P}(\mathcal{M})$ there exist a unique representative $\mathcal{Z} \in \mathfrak{Z}$ and inversely.

Proof. The proof of this proposition is based on the result provided in Lemma 2. For a clique $\mathcal{Z} \in \mathfrak{Z}$ it is straightforward 
to conclude that there is a unique $\mathcal{A} \in \mathcal{P}(\mathcal{M})$ that represent it. In fact, by the construction of the vertices $Z$ they satisfy constraint (11c). Further since $\mathcal{Z}$ is a clique then all vertices are connected, and hence they verify constraint (11b). For $\mathcal{A}=$ $\bigoplus_{Z \in \mathcal{Z}} Z$ the mapping is unique. Conversely, let $\mathcal{A}$ be a set of transmitting devices. From Lemma 2, there exist a unique $\mathcal{Z}$ that satisfy the constraints (11a), (11b), and (11c). Since all clusters $Z \in \mathcal{Z}$ satisfy (12) and (13), then they are generated in the graph and they are connected. In other words, $\mathcal{Z}$ is a clique which concludes the proof.

\section{Decoding Delay Reduction}

This subsection first reformulates the optimization problem in (4) in a more tractable form based on the results provided in Proposition 3. Afterwards, the problem is shown to be equivalent to a maximum weight clique problem in the full cooperation graph. Finally, the next subsection provides a method that relies on the generation of a well-defined set of clusters to construct the cooperation graph. Given the oneto-one mapping between the set of cliques and the set of transmitting devices, the optimization problem in (4) can be rewritten as follows:

$$
\begin{aligned}
& \max _{\mathcal{Z} \in \mathcal{Z}}-\left|\mathcal{Z} \cap M_{w}\right|-\left|\mathcal{T} \cap M_{w}\right|-\left|\mathcal{S} \cap M_{w}\right| \\
& +\sum_{Z \in \mathcal{Z}} \sum_{i \in Z}\left(\sum_{j \in \tau_{k}\left(\kappa_{i}^{*}\right)} 1-p_{i j}\right) \\
& \text { with } \kappa_{i}^{*}(\mathcal{Z})=\arg \max _{\kappa_{i} \in \mathcal{P}\left(\mathcal{H}_{i}\right)}\left(\sum_{j \in \tau_{i}\left(\kappa_{i}\right)} 1-p_{i j}\right)
\end{aligned}
$$

The following theorem characterizes the global optimal solution to the decoding delay reduction in IDNC-based D2D network (4):

Theorem 2. The optimal solution to the decoding delay reduction problem in partially connected IDNC-based D2D network (4) is equivalent to the maximum weight clique problem in the cooperation graph, in which the weight of each vertex $v$ representing the cluster $Z$ is:

$$
\begin{aligned}
v & =\left|\mathcal{C}^{T}(Z) \cap M_{w}\right|-\left|Z \cap M_{w}\right|-\left|\mathcal{T}(Z) \cap M_{w}\right| \\
& +\sum_{i \in Z}\left(\sum_{j \in \tau_{i}\left(\kappa_{i}^{*}\right)} 1-p_{i j}\right),
\end{aligned}
$$

where $\kappa_{k}^{*}$ is the maximum weight clique problem in the local IDNC graph of device $k$ for packet generation:

$$
\kappa_{i}^{*}=\arg \max _{\kappa \in \mathcal{G}_{i}}\left(\sum_{j \in \tau_{i}(\kappa)} 1-p_{i j}\right) .
$$

Proof. The proof of this theorem relies on the uniqueness of the clustering $\mathcal{Z}$ of each set of transmitting devices. Such mapping allows to reformulate the decoding delay reduction problem in terms of clusters of devices which can be expressed as as an optimization over the individual clusters $Z$. Finally, using the results of Theorem 1 , the proof concludes that the optimal solution to the optimization problem (14) is the maximum weight clique in the full cooperation graph. The complete proof can be found in Appendix F.

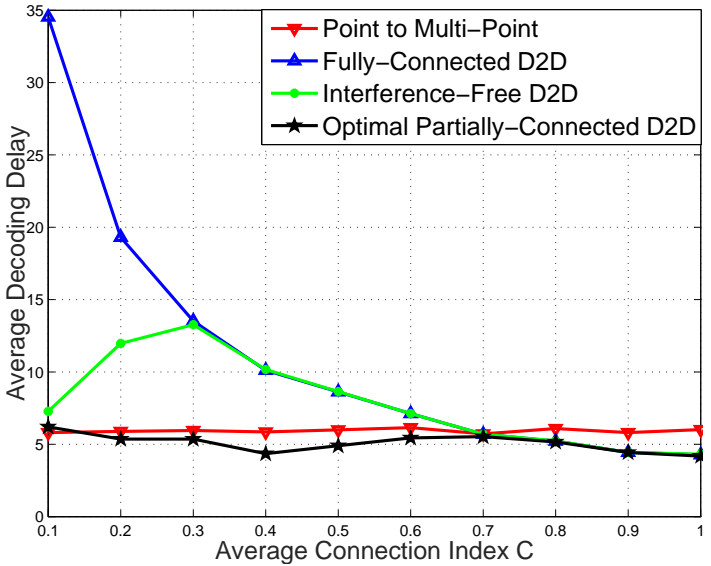

Fig. 2. Mean decoding delay versus the connectivity index $C$ for a network composed of $M=60$ devices, $N=30$ packets, an erasure probability $P=0.1$, and $Q=0.2$.

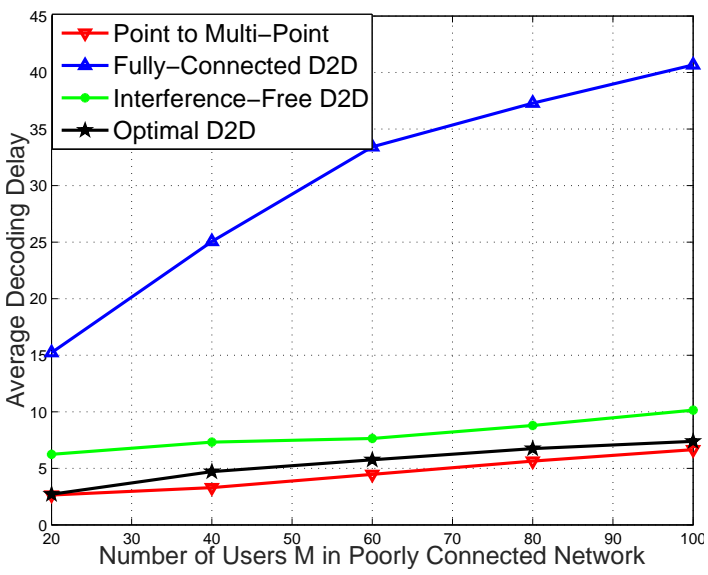

Fig. 3. Mean decoding delay versus number of devices $M$ for a network composed of $N=30$ packets, a connecitvity index $C=0.1$ an erasure probability $P=0.1$, and $Q=0.2$

\section{Simulation Results}

This section compares the average decoding delay experienced by a partially connected D2D system for the proposed collision-free and optimal schemes. As a base for comparison, the paper plots the decoding delay of a conventional PMP system [15], and the solution proposed in [30] for fully connected networks. The users are uniformly re-positioned for each iteration in a $500 \times 500 \mathrm{~m}$ grid with a connectivity radius $R$. The connectivity radius is chosen so as to obtain the required connectivity index $C$ defined as the ratio of the average number of neighbors by the total number of users $M$. In these simulations, the decoding delay is computed for 100 independent realizations, and the mean value is presented. Since the short range communications are more reliable than the base-station-to-device communications [30], the D2D packet erasure probability $P$ is set to be $P=Q / 2$ where $Q$ is the base-station-to-device packet erasure probability. These erasure probabilities are assumed to be perfectly known for all devices.

Fig. 2 depicts the comparison of the average decoding delay achieved by the PMP configuration, the fully connected D2D policy, and our partially connected D2D optimal and collisionfree policies against the connectivity index $C$ for $M=60$, 


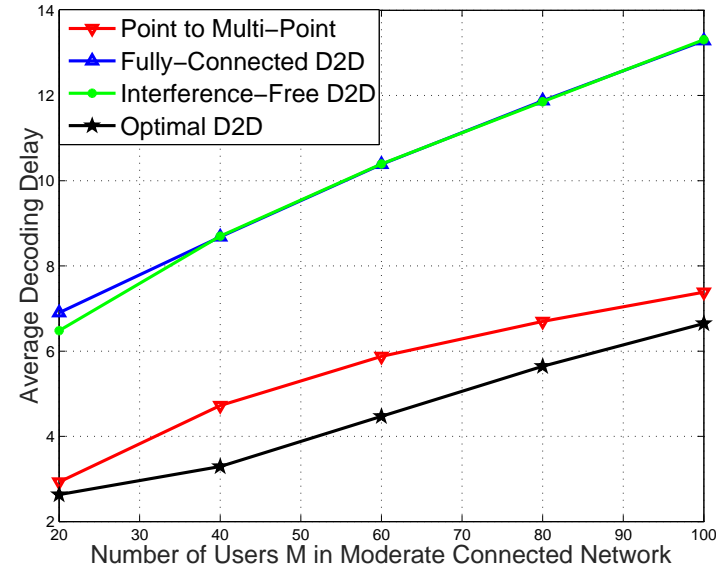

Fig. 4. Mean decoding delay versus number of devices $M$ for a network composed of $N=30$ packets, a connecitvity index $C=0.4$ an erasure probability $P=0.1$, and $Q=0.2$.

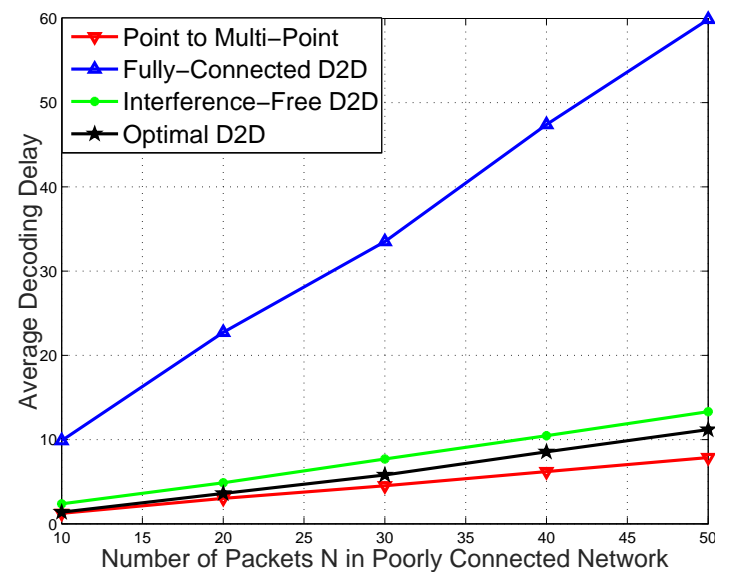

Fig. 5. Mean decoding delay versus number of packets $N$ for a network composed of $M=60$ devices, a connecitvity index $C=0.1$ an erasure probability $P=0.1$, and $Q=0.2$.

$N=30, P=0.1$ and $Q=0.2$. From Fig. 2, we clearly see that for a low connectivity index $(C \leq 0.3)$ our partially connected approach largely outperforms the fully connected approach. This can be explained by the fact that in the fully connected D2D, only one device is transmitting at each time slot. On the other hand, for a highly connected graph, a single device can target all others since the number of devices out of the transmission range is negligible. However for a low connectivity index, this term is no longer negligible. Further, the collision-free solution clearly degrades as the connectivity index increase. This can be explained by the fact that, as the connectivity index increases, the number of devices that can transmit simultaneously decreases and thus the scheme converges to the fully connected D2D.

Fig. 3 and Fig. 4 illustrate the average decoding delay achieved by the same policies against the number of devices $M$ for $N=30, P=0.1, Q=0.2$ for a poorly connected network $(C=0.1$ in Fig. 3) and a moderately connected network ( $C=0.4$ in Fig. 4). Fig. 5 and Fig. 6 show a similar comparison against the number of packets $N$ for $M=60$, $P=0.1, Q=0.2$ and $C=0.8$ for a poorly connected network $(C=0.1$ in Fig. 5) and a moderately connected network $(C=0.4$ in Fig. 6$)$.

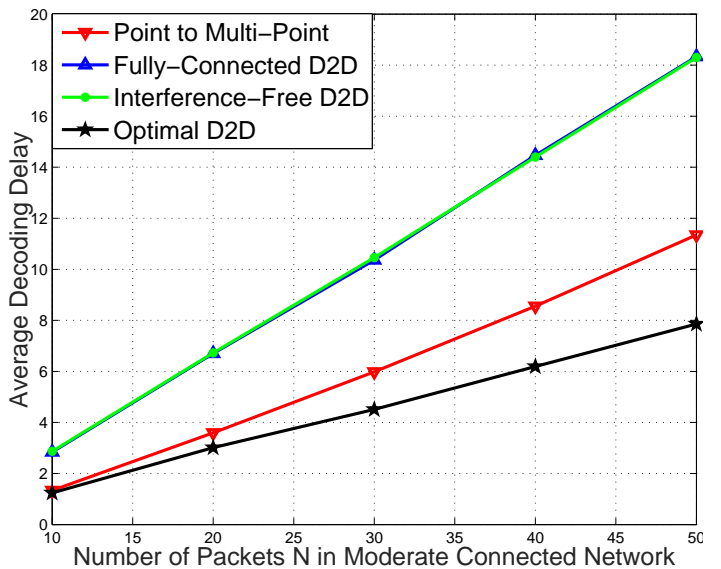

Fig. 6. Mean decoding delay versus number of packets $N$ for a network composed of $M=60$ devices, a connecitvity index $C=0.4$ an erasure probability $P=0.1$, and $Q=0.2$.

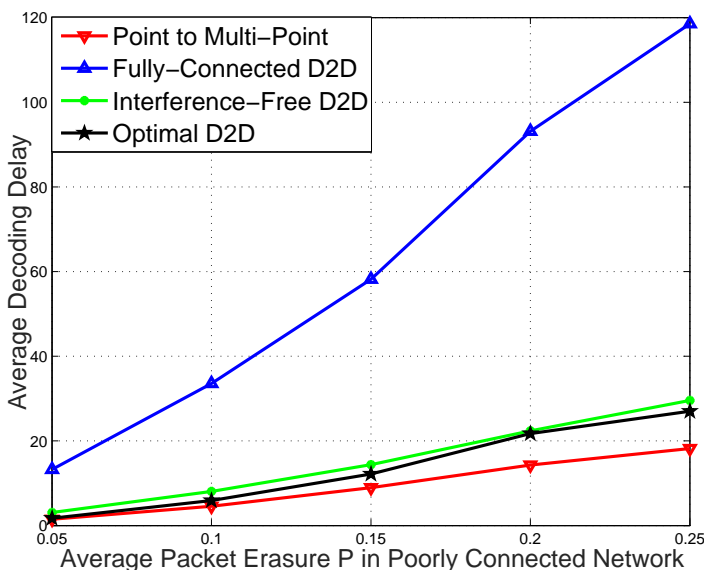

Fig. 7. Mean decoding delay versus erasure probability $P$ for a network composed of $M=60$ devices, $N=30$ packets, a connecitvity index $C=$ 0.1 an erasure probability $Q=2 P$.

For connectivity index $C=0.1$, the optimal and collisionfree partially connected policies perform closely as displayed in Fig. 3 and Fig. 5. This can be explained by the fact that, at low connectivity, the set of devices that can transmit simultaneously while respecting the non-collision constraint is large. Consequently, allowing collision does not provide a significant gain. Further, the partially connected algorithms provide an appreciable gain as compared with the fully connected solution as the number of devices and packets increases. For $C=0.4$, we can clearly from Fig. 4 and Fig. 6 see that the optimal partially connected policy outperforms the PMP approach. Note that the PMP approach performs better that the optimal D2D solution at low connectivity index, as it does not suffer from devices being out of the transmitting devices range and the delay encountered by the transmitting devices in the D2D case. As the connectivity index increases, these effects decrease in the D2D scenarios, thus enabling the optimal D2D approach to outperform the PMP as shown in Fig. 4 and Fig. 6.

Fig. 7 and Fig. 8 depict the same comparison for the decoding delay against the packet erasure probability $P$ for $M=60, N=30, Q=2 P, C=0.1$ for Fig. 7, and $C=0.4$ for Fig. 8. For a low connectivity index $C$, our proposed solutions outperform the fully connected one for all values of 


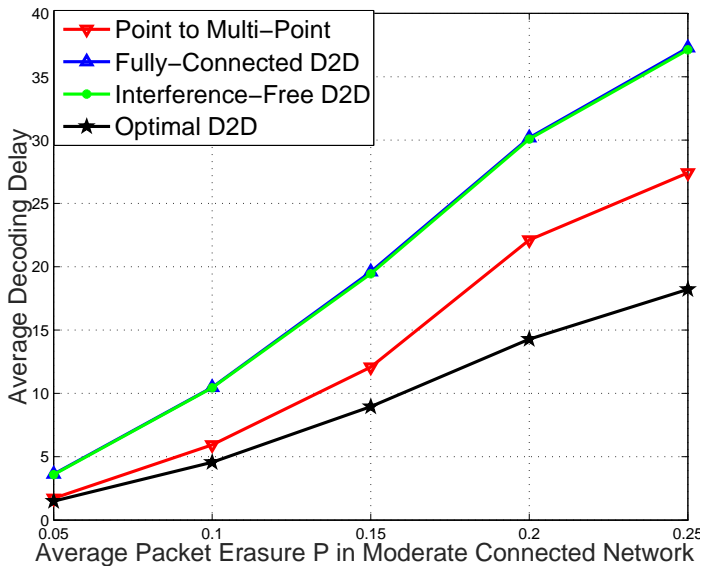

Fig. 8. Mean decoding delay versus erasure probability $P$ for a network composed of $M=60$ devices, $N=30$ packets, a connecitvity index $C=$ 0.4 an erasure probability $Q=2 P$.

erasure probabilities as shown in Fig. 7. As the connectivity index increases (Fig. 8), we clearly see that the performance of the optimal partially connected D2D is better than the PMP one. This can be clearly explained bu the fact that, as the erasure probabilities increase, the D2D setting provides a much better reachability and packet delivery compared to the PMP setting.

\section{CONCLUSION}

The paper investigates the decoding delay reduction problem in an IDNC-based device-to-device communications network. It considers a partially connected network in which more than one device can transmit simultaneously. The decoding delay minimization problem is formulated as a joint optimization over the set of transmitting devices and the packet combination to be transmitted. The paper introduces the local IDNC graph and the cooperation graph which enable the reformulation of the decoding delay reduction problem as a maximum weight clique search. Numerical results suggest that the PCD2D outperforms the FC-D2D and provides appreciable gains, especially for poorly connected networks. The results herein can be used in future research direction to propose an online completion time reduction strategy.

\section{APPENDIX A \\ Proof OF PROPOSITION 1}

From the definition of the decoding delay, device $i$, with non-empty Wants sets, experiences one unit increase of decoding delay if he cannot hear exactly one transmission or if he hears a packet that is either non-instantly decodable or non-innovative. The event of a device $i$, with non-empty Wants set, not be able to hear exactly one transmission occurs in the following scenarios:

- The device cannot hear any transmission: This event occurs if one of the following is true:

- The device is transmitting, i.e., $i \in \mathcal{A} \cap M_{w}$ ).

- The device is out of the transmission range of the transmitting devices, i.e., $i \in \mathcal{S} \cap M_{w}$.
- The device can hear multiple transmissions: This event occurs when the device is in the collision region of the transmitting devices, i.e., $i \in \mathcal{T} \cap M_{w}$.

The event of a device $i$, with non-empty Wants set, hearing a packet that is either non-instantly decodable or non-innovative happens when the device is in the opportunity zone of one of the transmitting devices, i.e., $i \in \mathcal{O}_{j}, j \in \mathcal{A}$, and one of the following event is true:

- He receives a non-innovative packet combination $\kappa_{j}$.

- He receives a non-instantly decodable packet combination $\kappa_{j}$.

These last two events translate the fact that device $i$ is not targeted by the transmission, i.e., $i \in \mathcal{O}_{j} \backslash \tau_{j}\left(\kappa_{j}\right)$. Due to the erasure nature of the links, the probability that the event occurs is $1-p_{j i}$.

In order to derive the expected decoding delay increase for an arbitrary device $i$ in the network, we first partition the set of devices.

Lemma 3. For any set of transmitting devices $\mathcal{A}$, the following sets forms a partition of the set of all devices $\mathcal{M}$ :

$$
\begin{aligned}
\mathcal{M}=\bigoplus_{i \in \mathcal{A}}\left(\mathcal{O}_{i} \cap M_{w}\right) \oplus\left(\mathcal{A} \cap M_{w}\right) \\
\oplus\left(\mathcal{T} \cap M_{w}\right) \oplus\left(\mathcal{S} \cap M_{w}\right) \oplus \bar{M}_{w} .
\end{aligned}
$$

Proof. We first prove that for any set of transmitting devices $\mathcal{A}$, the following results holds:

$$
\mathcal{M}=\bigcup_{i \in \mathcal{A}} \mathcal{O}_{i} \cup \mathcal{A} \cup \mathcal{T} \cup \mathcal{S} .
$$

Let $\mathcal{C}^{T}=\bigcup_{i \in \mathcal{A}} \mathcal{C}_{i}$ be the total coverage zone of the transmitting devices. By definition of the set $\mathcal{S}$, we have:

$$
\mathcal{M}=\mathcal{C}^{T} \cup \mathcal{S} \text {. }
$$

Hence the opportunity zone can be written as follows:

$$
\mathcal{O}_{i}=\mathcal{C}_{i} \backslash(\mathcal{A} \cup \mathcal{T}), \forall i \in \mathcal{A} .
$$

Developing the union of the opportunity zones yields:

$$
\begin{aligned}
\bigcup_{i \in \mathcal{A}} \mathcal{O}_{i} & =\bigcup_{i \in \mathcal{A}} \mathcal{C}_{i} \backslash(\mathcal{A} \cup \mathcal{T}) \\
\bigcup_{i \in \mathcal{A}} \mathcal{O}_{i} & =\mathcal{C}^{T} \backslash(\mathcal{A} \cup \mathcal{T}) \\
\mathcal{C}^{T} & =\bigcup_{i \in \mathcal{A}} \mathcal{O}_{i} \cup \mathcal{A} \cup \mathcal{T} .
\end{aligned}
$$

Therefore, we obtain the desired result:

$$
\mathcal{M}=\bigcup_{i \in \mathcal{A}} \mathcal{O}_{i} \cup \mathcal{A} \cup \mathcal{T} \cup \mathcal{S} .
$$

We now prove that the sets are disjoint. By definition of the sets $\mathcal{A}, \mathcal{T}$ and $\mathcal{S}$, we have that these sets are pairwise disjoint. Also by definition, we have the set $\mathcal{O}_{i}$ is disjoint from $\mathcal{A}$ and $\mathcal{T}, \forall i \in \mathcal{A}$. Finally, assume $\exists i \in \mathcal{O}_{j} \cap \mathcal{O}_{k}$ for $(j, k) \in$ $\mathcal{A}^{2}$. We have $\mathcal{O}_{i} \subset \mathcal{C}_{i}$. Therefore, $i \in \mathcal{C}_{j} \cap \mathcal{C}_{k}$. By definition of the collision set $\mathcal{T}$, we have $i \in \mathcal{T}$. By definition of the opportunity zone, we have $\mathcal{O}_{i} \cap \mathcal{T}=\varnothing, \forall i \in \mathcal{A}$. Hence such $i \in \mathcal{O}_{j} \cap \mathcal{O}_{k}$ does not exist and all sets $\mathcal{O}_{l}, \forall l \in \mathcal{A}$ are pairwise disjoint. Obviously, we have $(X \cap Y) \cap \bar{Y}=\varnothing, \forall X, Y$. This 
conclude our proof and we have:

$$
\begin{aligned}
\mathcal{M}=\bigoplus_{i \in \mathcal{A}} & \left(\mathcal{O}_{i} \cap M_{w}\right) \oplus\left(\mathcal{A} \cap M_{w}\right) \\
& \oplus\left(\mathcal{T} \cap M_{w}\right) \oplus\left(\mathcal{S} \cap M_{w}\right) \oplus \bar{M}_{w} .
\end{aligned}
$$

From Lemma 3, for any set of transmitting devices $\mathcal{A}$, the following sets forms a partition of the set of devices $\mathcal{M}$ :

$$
\begin{aligned}
\mathcal{M}=\bigoplus_{i \in \mathcal{A}} & \left(\mathcal{O}_{i} \cap M_{w}\right) \oplus\left(\mathcal{A} \cap M_{w}\right) \\
& \oplus\left(\mathcal{T} \cap M_{w}\right) \oplus\left(\mathcal{S} \cap M_{w}\right) \oplus \bar{M}_{w} .
\end{aligned}
$$

Given the partition of devices in (A.8) and the analysis above, the expected individual decoding delay $d_{j}(\mathcal{A}, \kappa)$ of device $j$ when devices in $\mathcal{A}$ are transmitting respectively the packets combinations $\kappa_{i}$ and $\kappa=\left\{\kappa_{i}\right\}_{i \in \mathcal{A}}$ is given by:

$$
\mathbb{P}\left(d_{j}(\mathcal{A}, \kappa)=1\right)= \begin{cases}0 & \text { if } j \in \bar{M}_{w} \\ 1 & \text { if } j \in \mathcal{A} \cap M_{w} \\ 1 & \text { if } j \in \mathcal{T} \cap M_{w} \\ 1 & \text { if } j \in \mathcal{S} \cap M_{w} \\ 0 & \text { if } j \in\left(\mathcal{O}_{i} \cap M_{w}\right) \cap \tau_{i}\left(\kappa_{i}\right) \\ 1-p_{i j} & \text { if } j \in\left(\mathcal{O}_{i} \cap M_{w}\right) \backslash \tau_{i}\left(\kappa_{i}\right) .\end{cases}
$$

Let $D(\mathcal{A}, \kappa)$ be the total decoding delay increase. From (A.8), all sets form a partition of $\mathcal{M}$ and thus are disjoint. Therefore, the expected overall decoding delay can be written as:

$$
\begin{aligned}
\mathbb{E}[D(\mathcal{A}, \kappa)]= & \sum_{i \in \mathcal{M}} d_{i}(\mathcal{A}, \kappa) \\
= & \left|\mathcal{A} \cap M_{w}\right|+\left|\mathcal{T} \cap M_{w}\right|+\left|\mathcal{S} \cap M_{w}\right| \\
& \quad+\sum_{i \in \mathcal{A}}\left(\sum_{j \in\left(\mathcal{O}_{i} \cap M_{w}\right) \backslash \tau_{i}\left(\kappa_{i}\right)} 1-p_{i j}\right) .
\end{aligned}
$$

Using the expected decoding delay increase expression (A.9), the decoding delay minimization problem can be formulated as the following joint optimization over the set of transmitting devices and the packet combination to be transmitted:

$$
\begin{aligned}
& \min _{\substack{\mathcal{A} \in \mathcal{P}(\mathcal{M}) \\
\kappa_{i}(\mathcal{A}) \in \mathcal{P}\left(\mathcal{H}_{i}\right)}} \mathbb{E}[D(\mathcal{A}, \kappa)] \\
& \Leftrightarrow \min _{\substack{\mathcal{A} \in \mathcal{P}(\mathcal{M}) \\
\kappa_{i} \in \mathcal{P}\left(\mathcal{H}_{i}\right)}}\left|\mathcal{A} \cap M_{w}\right|+\left|\mathcal{T} \cap M_{w}\right| \\
& \quad+\left|\mathcal{S} \cap M_{w}\right|+\sum_{i \in \mathcal{A}}\left(\sum_{\substack{j \in\left(\mathcal{O}_{i} \cap M_{w}\right) \backslash \tau_{i}\left(\kappa_{i}\right) \\
\Leftrightarrow}} 1-p_{i j}\right) \\
& \Leftrightarrow \max _{\substack{\mathcal{A} \in \mathcal{P}(\mathcal{M}) \\
\kappa_{i} \in \mathcal{P}\left(\mathcal{H}_{i}\right)}}-\left|\mathcal{A} \cap M_{w}\right|-\left|\mathcal{T} \cap M_{w}\right| \\
& \quad-\left|\mathcal{S} \cap M_{w}\right|+\sum_{i \in \mathcal{A}}\left(\sum_{j \in \tau_{i}\left(\kappa_{i}\right)} 1-p_{i j}\right) .
\end{aligned}
$$

Clearly, from the problem formulation (A), the packet combination that the transmitting devices can generate only affects the last term of the expression. Therefore, the decoding delay minimization problem can be reformulated as follows:

$$
\begin{aligned}
& \max _{\mathcal{A} \in \mathcal{P}(\mathcal{M})}-\left|\mathcal{A} \cap M_{w}\right|-\left|\mathcal{T} \cap M_{w}\right|-\left|\mathcal{S} \cap M_{w}\right| \\
& \quad+\sum_{i \in \mathcal{A}}\left(\sum_{j \in \tau_{i}\left(\kappa_{i}^{*}\right)} 1-p_{i j}\right),
\end{aligned}
$$

with the packet combinations being given by:

$$
\kappa_{i}^{*}=\arg \max _{\kappa_{i} \in \mathcal{P}\left(\mathcal{H}_{i}\right)}\left(\sum_{j \in \tau_{i}\left(\kappa_{i}\right)} 1-p_{i j}\right), \forall i \in \mathcal{A} .
$$

\section{APPENDIX B \\ PROOF OF LEMMA 1}

To proof this lemma, an approach similar to the one employed in [22] for a PMP network is used. Since a device $i$ can target only devices in his opportunity zone $\mathcal{O}_{i}$ and can make packet combination only using the packets it already holds, then the network can be reduced. In other words, considering the reduced network consisting of a set $\mathcal{M}^{\prime}=\mathcal{O}_{i}$ of devices and a set $\mathcal{N}^{\prime}=\mathcal{H}_{i}$ of packets yields the same solution. In fact, since $\tau_{i}\left(\kappa_{i}\right) \subseteq \mathcal{O}_{i}$, then the targeted devices in the original network are the same as in the reduced one. Moreover, given $\kappa_{i} \subseteq \mathcal{H}_{i}$ then the packet combination remains unchanged. Therefore, the problem can be expressed as:

$$
\kappa_{i}^{*}=\arg \max _{\kappa_{i} \in \mathcal{P}\left(\mathcal{N}^{\prime}\right)}\left(\sum_{j \in \tau_{i}\left(\kappa_{i}\right)} 1-p_{i j}\right) .
$$

According to the analysis done in [22], the optimization problem (B.1) is equivalent to a maximum weight clique in the IDNC graph [15] of the reduced network. Since the IDNC graph of the reduced network is equivalent to the local IDNC graph introduced in Section III, the result can be easily extended to the local IDNC graph. Therefore, the set of all feasible packet combinations in local IDNC is represented by all maximal cliques in $\mathcal{G}_{i}$. To generate a packet combination, binary XOR is applied to all packets identified by the vertices of a selected maximal clique $\kappa$ in $\mathcal{G}_{i}$. The targeted devices by this transmission $\kappa$ are those determined by the vertices of the selected maximal clique. In terms of the local IDNC graph, the optimization problem (5) is equivalent to:

$$
\begin{aligned}
\kappa_{i}^{*} & =\arg \max _{\kappa_{i} \in \mathcal{P}\left(\mathcal{H}_{i}\right)}\left(\sum_{j \in \tau_{i}\left(\kappa_{i}\right)} 1-p_{i j}\right) \\
& =\arg \max _{\kappa_{i} \in \mathcal{G}_{i}}\left(\sum_{j \in \tau_{i}(\kappa)} 1-p_{i j}\right), \forall i \in \mathcal{A} .
\end{aligned}
$$

where $\kappa$ is a clique in the local IDNC graph. Therefore, the solution of the optimization problem (5) for device $i \in \mathcal{A}$ is the maximum weight clique in the local IDNC graph $\mathcal{G}_{i}$ in which the weight of each vertex $v_{j l}$ is $1-p_{i j}$.

\section{APPENDIX C \\ PROOF OF LEMMA 2}

This proof establishes the independence of the set of transmitting devices and their packet combination given the collision-free constraint. Such conclusion is reached by showing that under the collision-free constraint, the local-IDNC 
graph of any transmitting user does not depend on the other transmitting device. Therefore, using the result of Proposition 1, one concludes the decoupling of the optimization problems (4) and (5). Finally, expanding the formulation of each problem allows to obtain the desired result.

Lemma 4. For any set of transmitting device $\mathcal{A} \in \mathcal{I}$ satisfying the non-collision constraint, we have:

$$
\mathcal{O}_{i}(\mathcal{A})=\mathcal{O}_{i}=\mathcal{C}_{i} \backslash\{i\} .
$$

Proof. Let $\mathcal{A} \in \mathcal{I}$ satisfying the non collision constraint, we have by definition of the collision set $\mathcal{T}(\mathcal{A})=\varnothing$. Hence, the expression of the opportunity zone $\mathcal{O}_{i}(\mathcal{A})$ of any device $i \in \mathcal{A}$ becomes:

$$
\begin{aligned}
\mathcal{O}_{i}(\mathcal{A}) & =\mathcal{C}_{i} \backslash(\mathcal{A} \cup \mathcal{T}(\mathcal{A})) \\
& =\mathcal{C}_{i} \backslash \mathcal{A} .
\end{aligned}
$$

We now prove that $\left(\mathcal{C}_{i} \backslash\{i\}\right) \cap \mathcal{A}=\varnothing$. Assume $\exists j \in$ $\left(\mathcal{C}_{i} \backslash\{i\}\right) \cap \mathcal{A}$. Then, by definition of the coverage zone, we have $j \in \mathcal{C}_{j}$ and thus $j \in \mathcal{C}_{j} \cap\left(\mathcal{C}_{i} \backslash\{i\}\right) \in \mathcal{T}(\mathcal{A})$. However, by definition of the set $\mathcal{A}$, the collision region is empty $\mathcal{T}(\mathcal{A})=$ $\varnothing$. Therefore, such $j \in\left(\mathcal{C}_{i} \backslash\{i\}\right) \cap \mathcal{A}$ does not exist, which conclude our proof and we have:

$$
\mathcal{O}_{i}(\mathcal{A})=\mathcal{O}_{i}=\mathcal{C}_{i} \backslash\{i\} .
$$

From Lemma 4, under the non-collision condition $\mathcal{T}=\varnothing$, the opportunity zone of any transmitting device $i$ is independent of the set of transmitting devices. In other words, for any set of transmitting device $\mathcal{A} \in \mathcal{I}$ :

$$
\mathcal{O}_{i}(\mathcal{A})=\mathcal{O}_{i}=\mathcal{C}_{i} \backslash\{i\} .
$$

The only variable that depends on the set of transmitting devices $\mathcal{A}$, in the construction of the local IDNC graph of device $i$, is the opportunity zone $\mathcal{O}_{i}$. Therefore, given that (C.4) holds under the non-collision constraint, then the local IDNC graph does not depend on the transmitting devices, i.e., $\mathcal{G}_{i}(\mathcal{A})=\mathcal{G}_{i}$. As a consequence, the optimal packet combination device $i$ can generate (optimization problem (5)) does not depend on the set of transmitting devices as showed in following equation:

$$
\begin{aligned}
\kappa_{i}^{*}(\mathcal{A}) & =\arg \max _{\kappa \in \mathcal{G}_{i}(\mathcal{A})}\left(\sum_{j \in \tau_{i}(\kappa)} 1-p_{i j}\right) \\
& =\arg \max _{\kappa \in \mathcal{G}_{i}}\left(\sum_{j \in \tau_{i}(\kappa)} 1-p_{i j}\right)=\kappa_{i}^{*}, \forall i \in \mathcal{A} .
\end{aligned}
$$

Let $y_{i}\left(\kappa_{i}^{*}\right)$ be the value of the objective function of (C.5). Note that $y_{i}\left(\kappa_{i}^{*}\right)$ is a non-negative function. The problem of selecting the transmitting devices can be expressed as:

$$
A^{*}=\arg \max _{\mathcal{A} \in \mathcal{I}}-\left|\mathcal{A} \cap M_{w}\right|-\left|\mathcal{S} \cap M_{w}\right|+\sum_{i \in \mathcal{A}} y_{i}\left(\kappa_{i}^{*}\right) .
$$

\section{APPENDIX D}

\section{PROOF OF THEOREM 1}

To prove this theorem, we first reformulate the optimization problem (8) in a more tractable form. Afterward, we show that there is a one-to-one mapping between the set of feasible transmitting devices and the cliques in the cooperation graph.
Finally, since the weight of the clique is equivalent to the objective function of (8) then the optimal solution of (8) is the maximum weight clique in the cooperation graph.

Let $\mathcal{C}^{T}(\mathcal{A})=\bigcup_{i \in \mathcal{A}} \mathcal{C}_{i}$ be the total coverage zone of the transmitting devices. The set of devices, with non-empty Wants set, out of the transmission range of the transmitting devices can be written, under the non-collision constraint, as follows:

$$
\begin{aligned}
\mathcal{S} \cap M_{w} & =\left(\mathcal{M} \backslash \mathcal{C}^{T}(\mathcal{A})\right) \cap M_{w}=\left(\mathcal{M} \cap \overline{\mathcal{C}^{T}}(\mathcal{A})\right) \cap M_{w} \\
& =\overline{\mathcal{C}^{T}}(\mathcal{A}) \cap M_{w}=\left(\overline{\mathcal{C}^{T}}(\mathcal{A}) \cap M_{w}\right) \cup\left(M_{w} \cap \bar{M}_{w}\right) \\
& =M_{w} \cap\left(\overline{\mathcal{C}^{T}}(\mathcal{A}) \cup \bar{M}_{w}\right)=M_{w} \cap\left(\overline{\mathcal{C}^{T}(\mathcal{A}) \cap M_{w}}\right) \\
& =M_{w} \backslash\left(\mathcal{C}^{T}(\mathcal{A}) \cap M_{w}\right) .
\end{aligned}
$$

Since $\mathcal{T}=\varnothing$, then we have $\mathcal{C}^{T}(\mathcal{A})=\bigoplus_{i \in \mathcal{A}} \mathcal{C}_{i}$. Therefore, the cardinality of the set $\mathcal{S} \cap M_{w}$ can be written as follows:

$$
\begin{aligned}
\left|\mathcal{S} \cap M_{w}\right| & =\left|M_{w}\right|-\left|\left(\bigoplus_{i \in \mathcal{A}} \mathcal{C}_{i} \cap M_{w}\right)\right| \\
& =\left|M_{w}\right|-\left|\bigoplus_{i \in \mathcal{A}}\left(\mathcal{C}_{i} \cap M_{w}\right)\right| \\
& =\left|M_{w}\right|-\sum_{i \in \mathcal{A}}\left|\mathcal{C}_{i} \cap M_{w}\right| .
\end{aligned}
$$

Note that, the first term in (D.2) is constant with respect to the set of transmitting devices and the transmitted packet combinations. Thus, this term is ignored in the optimization problem. Clearly, we have $\left|\mathcal{A} \cap M_{w}\right|=\sum_{i \in \mathcal{A}}\left|\{i\} \cap M_{w}\right|$. Hence, the optimization problem (8) can be reformulated as follows:

$$
A^{*}=\arg \max _{\mathcal{A} \in \mathcal{I}} \sum_{i \in \mathcal{A}}\left(\left|\mathcal{C}_{i} \cap M_{w}\right|-\left|\{i\} \cap M_{w}\right|+y_{i}\left(\kappa_{i}^{*}\right)\right)
$$

The rest of the section shown that there is a one-to-one mapping between the set of feasible transmitting devices, i.e., $\mathcal{A} \in \mathcal{I}$, and the set of cliques in the cooperation graph. Finally, to conclude the proof, we note that the weight of the clique is equivalent to the objective function of (D.3) which proves that the solution of the problem is equivalent to the maximum weight clique in the cooperation graph for a well-designed weights.

Let $\mathcal{A} \in \mathcal{I}$ be any combination of transmitting devices satisfying the non-collision constraint. We show that it can be represented by a clique $\kappa$ in the cooperation graph. Let $v_{i}$ be the vertices associated with the device $i \in \mathcal{A}$. By definition of $\mathcal{I}$ we have $\mathcal{C}_{i} \cap \mathcal{C}_{j}=\varnothing, \forall i \neq j \in \mathcal{A}$. Therefore, vertices $v_{i}$ and $v_{j}$ are connected which concludes that $\kappa$ is a clique in the cooperation graph.

In a similar way, let $\kappa$ be a clique in the cooperation graph associated with the set of transmitting devices $\mathcal{A}$. Since all nodes are pairwise connected then $\mathcal{C}_{i} \cap \mathcal{C}_{j}=\varnothing, \forall v_{i} \neq v_{j} \in$ $\kappa$ and hence $\mathcal{A} \in \mathcal{I}$. Therefore, there a one-to-one mapping between the set of feasible schedules $\mathcal{I}$ and the set of cliques in the cooperation graph. Finally, let $\kappa$ be a clique. The weight 


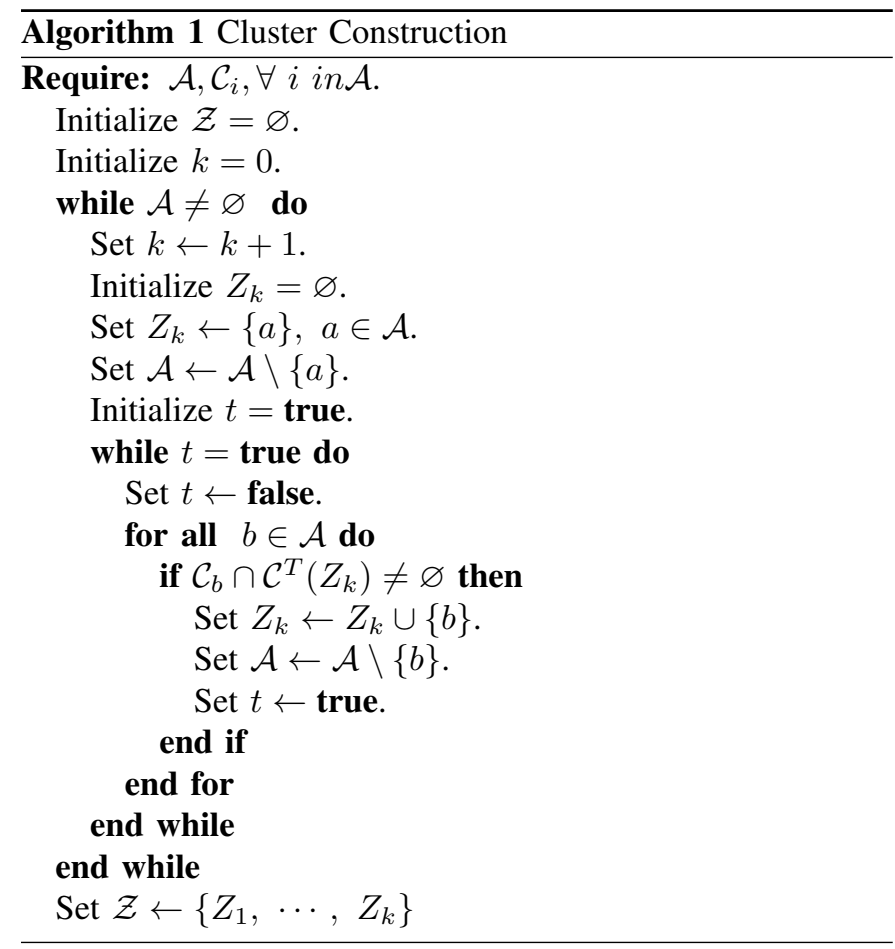

can be defined as follows:

$$
\begin{aligned}
w(\kappa) & =\sum_{v_{i} \in \kappa} w\left(v_{i}\right) \\
& =\sum_{v_{i} \in \kappa}\left(\left|\mathcal{C}_{i} \cap M_{w}\right|-\left|\{i\} \cap M_{w}\right|+y_{i}\left(\kappa_{i}^{*}\right)\right) .
\end{aligned}
$$

The weight in (D.4) is the objective function illustrated in (D.3). As a conclusion, the maximum weight clique in the cooperation graph is the optimal solution to the optimization problem (8) which conclude our proof.

\section{APPENDIX E}

\section{PROOF OF LEMMA 2}

In order to establishes the uniqueness of the decomposition, this proof first show the existence of the decomposition by suggesting a sequential constructing method. The proposed algorithm construct a new cluster of each vertex that does not satisfy all conditions (11a), (11b) and (11c). The second part of the proof shows that any other partition that satisfy all constraints can be reduced by means of permutations to the one reached by the proposed algorithm. In other words, the partition is unique.

To generate a clustering $\mathcal{Z}$ associated with the set of transmitting devices $\mathcal{A}$ respecting the conditions (11a), (11b) and (11c) simultaneously, this section proposes a sequential constructing method. A cluster $Z_{1}$ is first generated by including an arbitrary element of the set $\mathcal{A}$. Afterward, all remaining devices that are interfering with the cluster $Z_{1}$ are added to it. It is easy to see that such method of construction ensures that constraint (11c) holds. After this step, all remaining devices in $\mathcal{A}$ are not interfering with $Z_{1}$. A second cluster $Z_{2}$ is generated by including one of the transmitting devices in $\mathcal{A}$ and all interfering devices added to it. Note that all devices in $Z_{2}$ are not interfering with devices in $Z_{1}$. Therefore, $Z_{1}$ and $Z_{2}$ are not interfering and by extension the clustering $\mathcal{Z}$ satisfy the constraint (11b). The process is repeated until $\mathcal{A}=\varnothing$ which ensures that constraint (11a) is satisfied. Therefore, the algorithm proves the existence of a clustering $\mathcal{Z}$ respecting the conditions (11a), (11b) and (11c) simultaneously for all combination of transmitting devices $\mathcal{A}$. The steps of the algorithm are summarized in Algorithm 1.

Now we proof uniqueness of such decomposition. Let $\mathcal{A}$ be a set of transmitting devices and let $\mathcal{Z}$ and $\mathcal{Z}^{\prime}$ be two distinct clustering of the set $\mathcal{A}$ satisfying the constraints (11a), (11b) and (11c) simultaneously. From the constraint (11a), we have:

$$
\bigoplus_{Z \in \mathcal{Z}} Z=A=\bigoplus_{Z^{\prime} \in \mathcal{Z}^{\prime}} Z^{\prime}
$$

Note that this condition implies only that the clustering have the same number of devices and not necessarily the same clusters $Z$. Since $\mathcal{Z} \neq \mathcal{Z}^{\prime}$, given that both clustering satisfy (11a) then $\exists i \in Z \cap Z^{\prime}$ with $Z \in \mathcal{Z}, Z^{\prime} \in \mathcal{Z}^{\prime}, Z \neq Z^{\prime}$.

Let $K$ be the set constructed by first including the device $i$. Each device $k$ in the set $\mathcal{A}$ is included in $K$ if $\mathcal{C}_{k} \cap \mathcal{C}^{T}(K) \neq$ $\varnothing$. When no more devices can be added the process stop. Constraint (11b) can be written as:

$$
\mathcal{C}^{T}(Z) \cap \mathcal{C}^{T}(\mathcal{A} \backslash Z)=\varnothing, \forall Z \in \mathcal{A} \text {. }
$$

From (11b), we can clearly see that $K \subseteq Z$ and $K \subseteq Z^{\prime}$. Assume that $K \subset Z$, then from (11c), we have:

$$
K \subset Z \Leftrightarrow \mathcal{C}^{T}(Z \backslash K) \cap \mathcal{C}^{T}(K) \neq \varnothing .
$$

From the construction of the set $K$, we have:

$$
\mathcal{C}^{T}(A \backslash K) \cap \mathcal{C}^{T}(K)=\varnothing .
$$

Since $\mathcal{C}^{T}(Z \backslash K) \subset \mathcal{C}^{T}(A \backslash K)$, then $K=Z$. The same reasoning can be done to $Z^{\prime}$ and thus $Z=Z^{\prime}$ and $\mathcal{Z}=\mathcal{Z}^{\prime}$. This proves uniqueness of the decomposition and concludes our proof.

\section{APPENDIX F \\ PROOF OF THEOREM 2}

To prove this theorem, we first develop each term of the optimization problem (14) in order to reformulate the problem in a more tractable form. Exploiting the constraint in the creation of the clusters $Z \in \mathcal{Z}$, we show that the problem can be formulated as an optimization over the individual clusters $Z$. Finally, using the results of Theorem 1, the proof concludes that the optimal solution to the optimization problem (14) is the maximum weight clique in the full cooperation graph.

Using an analysis similar to the one done in (D.1), the set of devices out of the transmission range of the transmitting devices can be written as:

$$
\mathcal{S} \cap M_{w}=M_{w} \backslash\left(\mathcal{C}^{T}(\mathcal{Z}) \cap M_{w}\right) .
$$

Since $\mathcal{C}^{T}(Z) \cap \mathcal{C}^{T}\left(Z^{\prime}\right)=\varnothing, \forall Z \neq Z^{\prime} \in \mathcal{Z}$ (constraint (11b) on the generation of the clusters), then we can write $\mathcal{C}^{T}(\mathcal{Z})=\bigoplus_{Z \in \mathcal{Z}} \mathcal{C}^{T}(Z)$. Therefore, the cardinality of the set can be written as:

$$
\left|\mathcal{S} \cap M_{w}\right|=\left|M_{w}\right|-\sum_{Z \in \mathcal{Z}}\left|\mathcal{C}^{T}(Z) \cap M_{w}\right| .
$$

As for (D.2), the first term in (F.2) is constant. Therefore, it is removed from the optimization problem. Clearly, we have $\left|\mathcal{Z} \cap M_{w}\right|=\sum_{Z \in \mathcal{Z}}\left|Z \cap M_{w}\right|$. Similarly, the set of devices in 
collision can be reformulated using the clusters $Z$ as follows:

$$
\mathcal{T}(\mathcal{Z})=\bigcup_{Z, Z^{\prime} \in \mathcal{Z}} \bigcup_{\substack{z \in Z \\ z \neq z^{\prime} \in Z^{\prime}}}\left(\mathcal{C}_{z} \cap \mathcal{C}_{z^{\prime}}\right) .
$$

From the constraint (11b), inter-cluster collision region is empty. In other words, we have:

$$
\bigcup_{Z \neq Z^{\prime} \in \mathcal{Z}} \bigcup_{\substack{z \in Z \\ z^{\prime} \in Z^{\prime}}}\left(\mathcal{C}_{z} \cap \mathcal{C}_{z^{\prime}}\right)=\varnothing .
$$

Substituting (F.4) in (F.3) yields the following expression of the collision region:

$$
\begin{aligned}
\mathcal{T}(\mathcal{Z}) & =\bigcup_{Z \in \mathcal{Z}} \bigcup_{z \neq z^{\prime} \in Z}\left(\mathcal{C}_{z} \cap \mathcal{C}_{z^{\prime}}\right) \\
& =\bigoplus_{Z \in \mathcal{Z}} \mathcal{T}(Z) .
\end{aligned}
$$

Therefore, the cardinality of the set can be expressed as follows:

$$
\left|\mathcal{T} \cap M_{w}\right|=\sum_{Z \in \mathcal{Z}}\left|\mathcal{T}(Z) \cap M_{w}\right|
$$

Finally, the optimization problem (14) can be formulated as follows:

$$
\begin{gathered}
\mathcal{Z}^{*}=\arg \max _{\mathcal{Z} \in \mathcal{Z}} \sum_{Z \in \mathcal{Z}}\left|\mathcal{C}^{T}(Z) \cap M_{w}\right|-\left|Z \cap M_{w}\right| \\
-\left|\mathcal{T}(Z) \cap M_{w}\right|+y(Z)
\end{gathered}
$$

where the function $y(Z)$ is defined as follows:

$$
y(Z)=\sum_{i \in Z}\left[\max _{\kappa_{i} \in \mathcal{G}_{i}}\left(\sum_{j \in \tau_{i}\left(\kappa_{i}\right)}\left(1-p_{i j}\right)\right)\right]
$$

Since the clusters are non-interfering clusters, they can be seen as new devices in the network. Mirroring the steps used in Theorem 1, the optimal clustering $\mathcal{Z}^{*}$ is given by the maximal weight clique in the full cooperation graph where the weight of each vertex $v$ representing the cluster $Z$ can be expressed as:

$$
\begin{gathered}
v=\left|\mathcal{C}^{T}(Z) \cap M_{w}\right|-\left|Z \cap M_{w}\right|-\left|\mathcal{T}(Z) \cap M_{w}\right|+y(Z) . \\
\text { REFERENCES }
\end{gathered}
$$

[1] A. Douik, S. Sorour, T. Y. Al-Naffouri, H. C. Yang, and M. S. Alouini, "Delay reduction in multi-hop device-to-device communication using network coding," in Proc. of IEEE International Symposium on Network Coding (NetCod' 2015), Sydney, Australia, 2015, pp. 6-10.

[2] R. Ahlswede, N. Cai, S.-Y. Li, and R. Yeung, "Network information flow," IEEE Transactions on Information Theory, vol. 46, no. 4, pp. 1204-1216, Jul 2000.

[3] S. Sorour and S. Valaee, "On densifying coding opportunities in instantly decodable network coding graphs," in Proc. of IEEE International Symposium on Information Theory (ISIT' 2012), Cambridge, MA, USA, July 2012, pp. 2456-2460.

[4] T. Ho, R. Koetter, M. Medard, D. Karger, and M. Effros, "The benefits of coding over routing in a randomized setting," in Proc. of IEEE International Symposium on Information Theory (ISIT' 2003), Yokohama, Japan, June 2003, pp. 442-448.

[5] J.-S. Park, M. Gerla, D. Lun, Y. Yi, and M. Medard, "Codecast: a network-coding-based ad hoc multicast protocol," IEEE Wireless Communications, vol. 13, no. 5, pp. 76-81, October 2006.

[6] S. Katti, D. Katabi, W. Hu, H. Rahul, and M. Medard, "The Importance of Being Opportunistic: Practical Network Coding for Wireless Environments," in Proc. of Annual Allerton Conference on Communication, Control and Computing (Allerton' 2005), Monticello, Illinois, USA, 2005, pp. 84-85.

[7] S. Katti, H. Rahul, W. Hu, D. Katabi, M. Médard, and J. Crowcroft, "Xors in the air: Practical wireless network coding," in Proc. of the Conference on Applications, Technologies, Architectures, and Protocols for Computer Communications (SIGCOMM' 2006), Pisa, Italy, 2006, pp. 243-254.
[8] X. Li, C.-C. Wang, and X. Lin, "On the capacity of immediatelydecodable coding schemes for wireless stored-video broadcast with hard deadline constraints," IEEE Journal on Selected Areas in Communications, vol. 29, no. 5, pp. 1094-1105, May 2011.

[9] D. Traskov, M. Medard, P. Sadeghi, and R. Koetter, "Joint scheduling and instantaneously decodable network coding," in Proc. of IEEE Global Telecommunications Conference (GLOBECOM' 2009), Honolulu, Hawaii, USA, Nov 2009, pp. 1-6.

[10] A. Douik, S. Sorour, M. S. Alouini, and T. Y. Al-Naffouri, "Completion time reduction in instantly decodable network coding through decoding delay control," in Proc. of IEEE Global Telecommunications Conference (GLOBECOM' 2014), Austin, Texas, USA, Dec 2014, pp. 5008-5013.

[11] A. Douik, S. Sorour, T. Y. Al-Naffouri, and M. S. Alouini, "Decodingdelay-controlled completion time reduction in instantly decodable network coding," IEEE Transactions on Vehicular Technology, vol. 66, no. 3, pp. 2756-2770, March 2017.

[12] M. Esmaeilzadeh and P. Sadeghi, "Optimizing completion delay in network coded systems over tdd erasure channels with memory," in Proc. of IEEE International Symposium on Communications and Information Technologies (ISCIT' 2012), Queensland, Australia, Oct 2012, pp. 883888.

[13] S. Y. El Rouayheb, M. A. R. Chaudhry, and A. Sprintson, "On the minimum number of transmissions in single-hop wireless coding networks," in Proc. of IEEE Information Theory Workshop (ITW' 2007), Bergen, Norway, Sept 2007, pp. 120-125.

[14] A. Douik, S. Sorour, M. S. Alouini, and T. Y. Al-Naffouri, "On minimizing the maximum broadcast decoding delay for instantly decodable network coding," in Proc. of IEEE Vehicular Technology Conference (VTC-Fall' 2014), Vancouver, BC, Canada, Sept 2014, pp. 1-5.

[15] S. Sorour and S. Valaee, "Minimum broadcast decoding delay for generalized instantly decodable network coding," in Proc. of IEEE Global Telecommunications Conference (GLOBECOM' 2010), Miami, Florida, USA, Dec 2010, pp. 1-5.

[16] A. Douik, S. Sorour, T. Y. Al-Naffouri, and M.-S. Alouini, "Instantly decodable network coding for real-time device-to-device communications," EURASIP Journal on Advances in Signal Processing, vol. 2016, no. 1, pp. 1-12, Jan 2016.

[17] S. Sorour, A. Douik, S. Valaee, T. Al-Naffouri, and M. Alouini, "Partially blind instantly decodable network codes for lossy feedback environment," IEEE Transactions on Wireless Communications, vol. 13, no. 9, pp. 4871-4883, Sept 2014.

[18] A. Douik, S. Sorour, T. Y. Al-Naffouri, and M. S. Alouini, "Rate aware instantly decodable network codes," IEEE Transactions on Wireless Communications, vol. 16, no. 2, pp. 998-1011, Feb 2017.

[19] P. Sadeghi, D. Traskov, and R. Koetter, "Adaptive network coding for broadcast channels," in Proc. of Workshop on Network Coding, Theory, and Applications (NetCod' 2009), Lausanne, Switzerland, June 2009, pp. 80-85.

[20] A. Douik, S. Sorour, T. Y. Al-Naffouri, and M.-S. Alouini, "Delay reduction for instantly decodable network coding in persistent channels with feedback imperfections," IEEE Transactions on Wireless Communications, vol. 14, no. 11, pp. 5956-5970, 2015.

[21] L. Lu, M. Xiao, and L. K. Rasmussen, "Design and analysis of relayaided broadcast using binary network codes," Journal of Communications, vol. 6, no. 8, pp. 610-617, 2011.

[22] A. Le, A. Tehrani, A. Dimakis, and A. Markopoulou, "Instantly decodable network codes for real-time applications," in Proc of International Symposium on Network Coding (NetCod'2013), Calgary, Canada, June 2013, pp. 1-6.

[23] A. Douik, S. Sorour, T. Y. Al-Naffouri, and M. S. Alouini, "Instantly decodable network coding: From centralized to device-to-device communications," IEEE Communications Surveys Tutorials, vol. 19, no. 2, pp. 1201-1224, Secondquarter 2017.

[24] A. Douik and S. Sorour, "Data dissemination using instantly decodable binary codes in fog-radio access networks," IEEE Transactions on Communications, early access, 2018.

[25] A. Tehrani and A. Dimakis, "Finding three transmissions is hard," in Proc. of IEEE Global Telecommunications Conference (GLOBECOM' 2012), Anaheim, California, USA, Dec 2012, pp. 2293-2298.

[26] E. Drinea, C. Fragouli, and L. Keller, "Delay with network coding and feedback," in Proc. of IEEE International Symposium on Information Theory (ISIT' 2009), Seoul, Korea, June 2009, pp. 844-848.

[27] Q. Wu, G. Y. Li, W. Chen, D. W. K. Ng, and R. Schober, "An overview of sustainable green 5G networks," IEEE Wireless Communications, vol. 24, no. 4, pp. 72-80, 2017.

[28] Q. Wu, G. Y. Li, W. Chen, and D. W. K. Ng, "Energy-efficient $\mathrm{d} 2 \mathrm{~d}$ overlaying communications with spectrum-power trading," IEEE 
Transactions on Wireless Communications, vol. 16, no. 7, pp. 44044419, 2017.

[29] S. Zhang, Q. Wu, S. Xu, and G. Y. Li, "Fundamental green tradeoffs: Progresses, challenges, and impacts on 5G networks," IEEE Communications Surveys \& Tutorials, vol. 19, no. 1, pp. 33-56, 2017.

[30] N. Aboutorab, P. Sadeghi, and S. Tajbakhsh, "Instantly decodable network coding for delay reduction in cooperative data exchange systems," in Proc. of IEEE International Symposium on Information Theory (ISIT' 2013), Istanbul, Turkey, July 2013, pp. 3095-3099.

[31] M. Karim, N. Aboutorab, A. Nasir, and P. Sadeghi, "Decoding delay reduction in network coded cooperative systems with intermittent status update," in Proc. of IEEE Information Theory Workshop (ITW' 2014), Hobart, Tasmania, Australia, Nov 2014, pp. 391-395.

[32] A. Douik, S. Sorour, H. Tembine, M. S. Alouini, and T. Y. AlNaffouri, "A game theoretic approach to minimize the completion time of network coded cooperative data exchange," in Proc. of IEEE Global Telecommunications Conference (GLOBECOM' 2014), Austin, Texas, USA, Dec 2014, pp. 1583-1589.

[33] A. Douik, S. Sorour, H. Tembine, T. Y. Al-Naffouri, and M. S. Alouini, "A game-theoretic framework for network coding based device-to-device communications," IEEE Transactions on Mobile Computing, vol. 16, no. 4, pp. 901-917, April 2017.

[34] P. R. J. Ostergard, "A fast algorithm for the maximum clique problem," Discrete Appl. Math, vol. 120, pp. 197-207, 2002.

[35] K. Yamaguchi and S. Masuda, "A new exact algorithm for the maximum weight clique problem," in Proc. Of the 23rd International Technical Conference on Circuits/Systems, Computers and Communications (ITCCSCC' 2008), Yamaguchi, Japan, 2008, pp. 317-320.

[36] N. Bourgeois, B. Escoffier, V. T. Paschos, and J. M. van Rooij, "A bottom-up method and fast algorithms for max independent set," in Proc. of the 12th Scandinavian Conference on Algorithm Theory (SWAT' 2010), Bergen, Norway. Springer, 2010, pp. 62-73. 